\title{
28 Research Square \\ Congenital Bilateral and Multifocal Mesenchymal hamartoma of the chest wall: A Case in a Neonate and Differential Diagnosis
}

jiwei li

Kunming Children's Hospital https://orcid.org/0000-0002-1903-0731

Tie song Zhang

Kunming Children's Hospital

li Li

Kunming Children's Hospital

Meifen Wang

Kunming Children's Hospital

lan shen ( $\nabla 78801526 @ q q . c o m$ )

First People's Hospital of Honghe State

\section{Case Report}

Keywords: Mesenchymal hamartoma, Bilateral, Multifocal, Fetus

Posted Date: February 19th, 2021

DOI: https://doi.org/10.21203/rs.3.rs-248072/v1

License: (c) (i) This work is licensed under a Creative Commons Attribution 4.0 International License. Read Full License 


\section{Abstract}

\section{Background:}

Mesenchymal hamartoma of the chest wall(MHCW) is a rare benign extrapleural lesion that arises from rib or spine of neonates or early infancy, and commonly presents as a unilateral mass. Here, we describe a rare case of bilateral and multifocal MHCW in the right 5th rib and the left 11-12th thoracic and 1st lumbar vertebrae.

\section{Case presentation:}

A male neonate of $3700 \mathrm{~g}$ weight transferred to our hospital with mild respiratory distress and an asymptomatic, progressively enlarging intra-thoracic mass. Computed tomography scan(CT) and magnetic resonance imaging(MRI) indicated a large well-circumscribed, heterogeneous enhancing mass, arising from the posterior-medial aspect of the right chest wall, involving 3-5th posterior ribs. Other smaller sizes lesions observed in vertebral arch and transverse process of the left 11-12th thoracic and 1 st lumbar vertebrae. The thoracotomy with en bloc resection of the mass and the 5th rib was performed. Microscopically, the lesion was cystic and solid, consisted of hyaline cartilages, mesenchymal cells, and various-sized hemorrhagic spaces. Immunohistochemically, the cartilages and mesenchymal cells were positive for S-100, Smooth Muscle Actin(SMA), CD163 and CD68. The epithelium cells of aneurysmal bone cyst(ABC)-like were negative for CD34. Ki-67 estimated at 5\%-10\%. Fluorescence in situ hybridization(FISH) was negative for USP6 gene break-apart probe, which ruled out primary ABC. At 8month follow-up postoperatively, the right chest walls with no sign of recurrence and the lesions of left vertebral arch and transverse process were stable, no increased in size.

\section{Conclusion:}

The bilateral and multifocal MHCW is considerably rare and related clinical research is limited. Fortunately, we clearly observed the tumor's origin, growth process from the earlier pregnancy to delivery, different effects during the fetal periods, and differential diagnosis. This report may raise awareness regarding the MHCW.

\section{Background}

Mesenchymal hamartoma of the chest wall(MHCW), also known as chondromesenchymal hamartoma, is a rare benign extrapleural lesion that arises from rib ${ }^{[1]}$. It has an incidence of about $0.03 \%$ among primary bone tumors ${ }^{[2,3]}$, and mostly occurs in fetal life, neonates or early infancy ${ }^{[4]}$. Multifocal or/and bilateral lesions are extremely rare. Herein, we present the imaging and pathological features, treatment and prognosis of a male neonate with bilateral and multifocal MHCW, and a brief review of the literature.

\section{Case Presentation}


A 30-year-old pregnant woman gravida 2 para 1 at the 22nd gestation week, accepted routine pregnancy US at a local hospital. Examination showed a fetus of a weight of $744 \mathrm{~g}$ and head-first presentation and normal amniotic fluid volume. Moreover, there was a hyper-echoic mass of $14 \times 11 \mathrm{~mm}$ at the right posterior chest wall of the fetus (Fig. 1A). Afterwards, the woman transferred to a local 3A hospital, accepted fetal Ultrasound(US) and MRI at the 26th gestation week. US showed a fetus of a weight of $1113 \mathrm{~g} \pm 162 \mathrm{~g}$ with a heterogeneous hyper-echoic mass of $24 \times 12 \mathrm{~mm}$ situated at the posterior $4-5$ th ribs of the right posterior-medial chest wall(Fig. 1B). MRI showed a well-defined lobulated, mixed mass of $19 \times 16 \mathrm{~mm}$ at lower lobe of right lung. Both radiologist and obstetrician more considered that this mass was a teratoma or neurogenic tumor, and suggested the woman reviewing periodically. At the 37th gestation week, the woman delivered a male baby of $3700 \mathrm{~g}$ weight and the medical examination was normal except for mild respiratory distress. The neonate transferred to our hospital with an asymptomatic, progressively enlarging intra-thoracic mass. CT showed a large well-circumscribed, heterogeneous enhancing mass, measuring approximately $56 \times 50 \mathrm{~mm}$, with several speckled and multiple strip vascular internal shadows, arising from the posterior-medial aspect of the right chest wall, involving 3-5th posterior ribs(Fig. 1C). The cortical and medullary of 5th rib were observed the destructive-expansile changes. The 3-4th ribs were compressed and deformed. Other lesions of similar nature but of smaller sizes were also observed in vertebral arch and transverse process of the left 11-12th thoracic and 1st lumbar vertebrae(Fig. 1D). MRI showed that the mass was solid-cystic with multiple fluid-fluid levels(Fig. 1E and 1F), which was suspected to be a benign chondrogenic tumor. A month later, the baby underwent thoracotomy with en bloc resection of the mass and the 5th rib. Furthermore, any evidence of invasion or metastatic disease by the mass have been not observed. Gross examinations showed a grayred mass connected to partial rib with unclear boundary, measuring approximately

$75 \times 41 \times 35 \mathrm{~mm}$ (Fig. $2 \mathrm{~A}$ ). The cut surface was solid-cystic, gray white and dark red. The solid area was gray white, soft to hard. The cystic area was various-sized cysts with dark red liquid flowing out(Fig. 2B). Microscopically, the solid area consisted of mature or immature irregular hypercellular hyaline cartilages with foci of endochondral calcification and ossification, isolated by fascicles of immature spindle-shaped mesenchymal cells(Fig. 2C). Scattered woven bones were observed(Fig. 2D). The cystic area was composed of various-sized hemorrhagic spaces enclosed by fibrous cyst walls with multinucleated osteoclastic giant cells and scattered osteoid trabeculae, as secondary aneurysmal bone cyst(ABC)-like structures(Fig. 2C). Abnormal mitoses and atypia were not seen. Immunohistochemically, the cartilaginous areas were positive for S-100(Fig. 2E). Mesenchymal spindle-shaped cells were immunoreactive for SMA, CD163. Osteoclastic giant cells were positive for CD68. The epithelium cells of ABC-like were negative for CD34, but interstitial blood vessels were positivity(Fig. 2F). Ki-67 proliferation index was approximately 5\%-10\%. Fluorescence in situ hybridization(FISH) revealed the negativity of USP6 gene break-apart probe(Fig. 2G), which confirmed the final diagnosis of MHCW and ruled out primary ABC. Subsequently, the thoracic tube drain was removed in the 7th day after surgery(Fig. 1G), and the infant was discharged from the hospital in the 2nd postoperative week. Post-operative follow-up at one month and six months showed the patient to be developmentally well. CT scan at 6 months postoperatively showed the right chest walls with no sign of recurrence and a normal lung appearance, and the lesions of left vertebral arch and transverse process were stable, no increased in size(Fig. $1 \mathrm{H})$. 


\section{Discussion And Conclusions}

$\mathrm{MHCW}$ is a rare benign nature lesion composed of non-neoplastic skeletal tissues, with incidence of less than 1 per million in the general population ${ }^{[5]}$. It commonly presents as unilateral mass and locates in the right thoracic cavity ${ }^{[6]}$. Occasionally a few cases of multifocal and/or bilateral lesions have been reported $[4,7-10]$, only 5 multifocal cases and 10 bilateral cases in the previous literatures. In terms of our case, we clearly observed the growth of MHCW from the earlier pregnancy to delivery, obviously, the tumor was congenital and had different effects during the fetal periods. In the earlier days, it was too small to be found and to affect the lungs, thorax, spine. As the tumor grew larger and multifocal, it gradually compressed the lung and caused deformation of the ribs and spine, especially in the late fetal period. Subsequently, in neonate, the tumor presented the clinical symptoms as the results of long term occupancy and destruction: respiratory distress, cough, fever, scoliosis, and chest wall deformity. Therefore, we considered that MHCW gradually evolved into a diverse structure from the fetus, and mainly affected neonate and early infant.

In the diagnosis of MHCW, different imaging methods have their own advantages and disadvantages. On US, it is very helpful and useful in early detecting the site, size, density, enlargement, adjacent structures and margin, but it can't differentiate the origin from rib or soft tissue, benign or malignant ${ }^{[11,12]}$. CT scan clearly reveals the extrapleural origin, osteoid, chondroid, osseous expansion and destruction, and hemorrhagic cystic regions ${ }^{[13]}$, but which can't make differential diagnosis with malignant chest wall tumors such as Ewing sarcoma, rhabdomyosarcoma, osteosarcoma, chondrosarcoma or metastasis of leukemia, neuroblastoma and lymphoma ${ }^{[14]}$. MRI also observes the characteristics of MHCW like CT, especially in ABC-like fluid-fluid levels, but it is difficult to distinguish between the primary and secondary $A B C^{[7]}$.

It is noteworthy that MHCW is commonly accompanied with the ABC-like structures ${ }^{[15]}$, which easily makes misdiagnosis with primary ABC. Immunohistochemical markers such as S-100,SMA,CD68,CD163 and CD34, all of those substantiate the characteristics of histological diversity of MHCW. Furthermore, USP6 rearrangement is found in almost $69 \%$ of primary $A B C^{[16]}$, but not in secondary $A B C$ of $M H C W$.

According to the literatures, there are two main treatment approaches for MHCW. For the patients of unilateral and larger volume of $\mathrm{MHCW}$, with clinical respiratory symptoms, the surgical resection is a very suitable treatment ${ }^{[4]}$. For the smaller asymptomatic patients, the conservative management with imaging modality is recommended ${ }^{[17]}$. The prognosis of most patients is favorable ${ }^{[18]}$, only a few cases have recurrence at the post-operative period, and even one with malign transformation ${ }^{[19]}$. In our case, we chose the surgical resection for right thoracic lesion in the 5th rib, and the conservative management for the smaller sizes lesions in the vertebral arch and transverse process of the left 11-12th thoracic and 1st lumbar vertebrae. By post-operative follow-up and imaging modality, we didn't observe the sign of recurrence, and the other lesions were stable and stopped growing. The prognosis was good and consistent with a few literatures about multifocal lesions ${ }^{[20,21]}$. We will keep following up. 
In conclusion, $\mathrm{MHCW}$ is a rare congenital benign lesion that usually originates from the rib of the fetus, and presents the clinical symptoms in neonates or early infancy. It is extremely rare to observe the multifocal and/or bilateral lesions. This report may raise awareness regarding the presentation, diagnosis, and treatment of MHCW among pathologists, radiologists, and clinicians.

\section{Abbreviations}

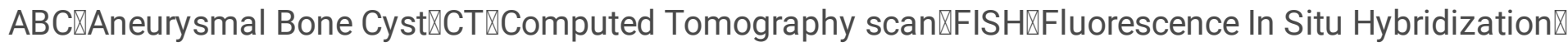

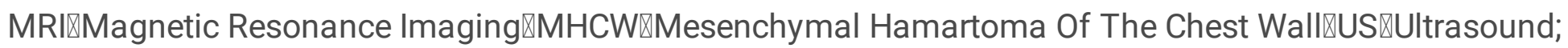
WHO $₫$ World Health Organization $₫ S M A$ :Smooth Muscle Actin

\section{Declarations}

\section{Acknowledgements}

We would like to thank Yangyang Ma for English language editing.

\section{Authors' contributions}

$\mathrm{JL}$ and LS was a major contributor in writing the manuscript and concept of the manuscript. TZ and LL revised manuscript as well as guidance and editing throughout the writing process. MW performed the follow-up.

\section{Funding}

Kunming health and Family Planning Commission Project:2019-SW-33

Yunnan Province's reserve medical talents project囚H-2019002

Kunming Medical University Applied Basic Research Joint Special Project:202001AY070001-170

\section{Availability of data and materials}

The data used and/or analyzed during the current study are available from the first author on reasonable request.

\section{Ethics approval and consent to participate}

The case report protocol was approved by the institutional ethics and research review boards at Kunming Children's Hospital. Patients signed a written informed consent upon admission to the hospital.

\section{Consent for publication}

Written informed consent was obtained from the patients for the publication of this case report and any accompanying images. A copy of the consent form is available for review by the Editor of this journal. 


\section{Competing interests}

The authors declare that they have no competing interests.

\section{Author details}

${ }^{1}$ Department of Pathology, ${ }^{2,3}$ Kunming Key Laboratory of Children Infection and Immunity, ${ }^{4}$ Department of Gastroenterology, Kunming Children's Hospital, Kunming 650228, Yunnan Province, China.

${ }^{5}$ Department of Emergency, First People's Hospital of Honghe State, Honghe State 661000, Yunnan Province, China.

${ }^{*}$ Corresponding author:

Lan Shen, Department of Emergency, First People's Hospital of Honghe State, Honghe State, China. Tel: (86) 873-6372-0121; Fax: (86) 873-6372-0277; E-mail: 78801526@qq.com

\section{References}

1. Okamoto K, Tani Y, Yamaguchi T, et al. Asymptomatic mesenchymal hamartoma of the chest wall in child with fluorodeoxyglucose uptake on PET/CT-Report of a case.[J]. Int Surg. 2015;100:915-9.

2. Braatz B, Evans R, Kelman A, Cheng W. Perinatal evolution of mesenchymal hamartoma of the chest wall.[. J]Pediatr Surg. 2010;45(12):e37-40.

3. Amstalden EM, Carvalho RB, Pacheco EM, Oliveira-Filho A, Stragea-Neto L, Rosenberg AE. Chondromatous hamartoma of the chest wall: description of 3 new cases and literature review.[J]. Int J Surg Pathol. 2006;14:119-26.

4. Alfaraidi M, Alaradati $\mathrm{H}$, Mamoun I, Mohammed S. Bilateral Mesenchymal Hamartoma of the Chest Wall in a 3-month-old boy: a case report and review of the literature.[J]. Case Rep Pathol,2017,(20178-16), 2017:1-4.

5. Richard DCD, McLeod A. Hamartoma (Mesenchymoma) of the Chest Wall in infancy.[J]. Radiology. 1979;131(3):657-61.

6. Haase R, Merkel N, Milzsch M, Lieser U. Mesenchymal chest wall hamartoma surgery is preferred.[J]. Arch Perinat Med. 2007;13:56-61.

7. Altaner S, Sodhi KS, Menon P, Dey P, Khandelwal N. Multifocal mesenchymal hamartoma of the chest wall.[J]. Respirology. 2006;11:334-8.

8. Li R, Kelly D, Siegal GP. Bilateral mesenchymal hamartoma of the chest wall in an infant boy.[J]. Fetal Pediatr Pathol. 2012;31(6):415-22.

9. Troum S, Dalton ML, Donner RS, Besser AS. Multifocal mesenchymal hamartoma of the chest wall in infancy.[J]. J Pediatr Surg. 1996;31(5):713-5.

10. Sodhi KS, Aiyappan SK, Menon P, Dey P, Khandelwal N. Unilateral multifocal mesenchymal hamartoma of the chest wall: a case report and review of literature.[J]. J Pediatr Surg. 
2009;44(2):464-7.

11. Yeshvanth SK, Shivamurthy V, Patil C, Rai S, Lakshminarayana KP, Makannavar JH. Mesenchymal hamartoma of the chest wall- mimicker of malignancy.[J]. J Cancer Res Ther. 2011;7(4):496-8.

12. Kim JY, Jung WH, Yoon CS, Kim MJ, Kim HK, Kim KD, Cho SH, et al. Mesenchymal hamartomas of the chest wall in infancy: radiologic and pathologic correlation.[J]. Yonsei Med J. 2000;41(5):61522.

13. Brand T, Hatch El, Schaller RT, et al. Surgical management of the infant with mesenchymal hamartoma of the chest wall.[J]. J Pediatr Surg. 1986;21(6):556-58.

14. Groom KR, Murphey MD, Howard LM, et al. Mesenchymal hamartoma of the chest wall: radiologic manifestations with emphasis on cross-sectional imaging and histopathology comparison.[J]. Radiology. 2002;222(1):205-11.

15. Rădulescu R, Bădilă A, Manolescu R, Sajin M, Japie I. Aneurysmal bone cyst clinical and morphological aspects.[J]. Romanian J Morphol Embryol. 2014;55(3):977-81.

16. Oliveira AM, Perez-Atayde AR, Inwards CY, Medeiros F, Derr V, Hsi BL, Gebhardt MC, Rosenberg AE, Fletcher JA. USP6 and CDH11 oncogenes identify the neoplastic cell in primary aneurysmal bone cysts and are absent in so-called secondary aneurysmal bone cysts.[J]. Am J Pathol. 2004;165(4):1773-80.

17. Jozaghi Y, Emil S, Albuquerque P, et al. Prenatal and postnatal features of mesenchymal hamartoma of the chest wall: Case report and literature review.[J]. Pediatr Surg Int. 2013;29(7):735-40.

18. Cameron D, Ong TH, Borzi P. Conservative management of mesenchymal hamartomas of the chest wall.[J]. J Pediatr Surg. 2001;36(9):1346-9.

19. Dounies R, Chwals WJ, Lally KP, et al. Hamartomas of the chest wall in infants.[J]. Ann Thorac Surg. 1994;57(4):868-75.

20. Freeburn AM, McAloon J. Infantile chest hamartoma case outcome aged 11.[J]. Arch Dis Child. 2001;85(3):244-5.

21. Kolar M, Pilkington $M$, Silva $M$, et al. Nonsurgical management of mesenchymal hamartomas of the chest wall.[J]. J Pediatr Surg Case Rep. 2018;39:17-21.

\section{Figures}




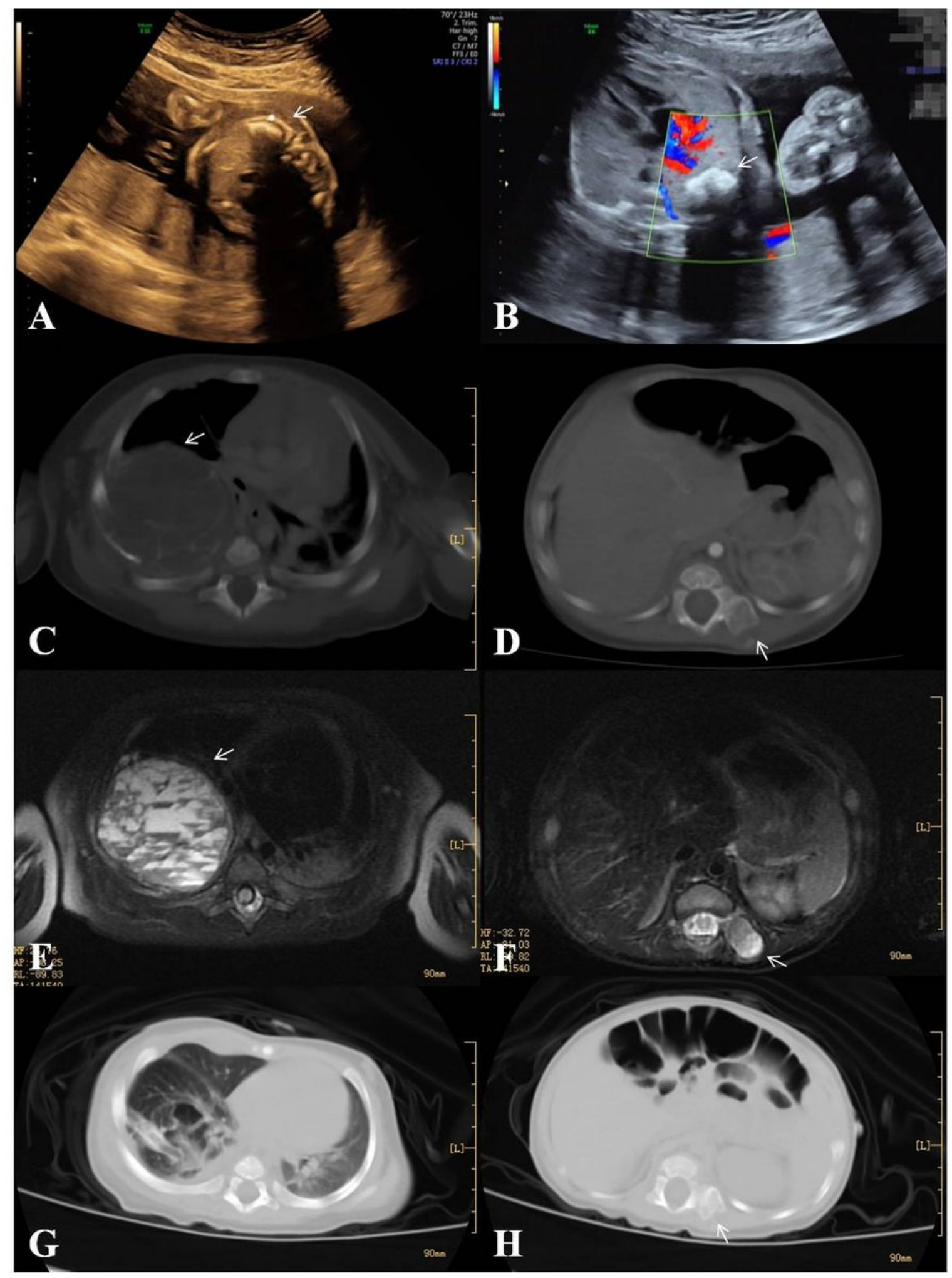

\section{Figure 1}

A The hyper-echoic mass at the 22nd gestation week(Arrow). B The hyper-echoic mass at the 26th gestation week(Arrow). C A large well-circumscribed, heterogeneous enhancing mass, and the destructiveexpansile ribs(Arrow). D Other expansile lesion(Arrow). E The mass with multiple fluid-fluid levels(Arrow). F The smaller lesion in the left vertebral arch and transverse process(Arrow). G Post-operative CT . H The lesions of left vertebral arch and transverse process at 6 months postoperatively(Arrow). 


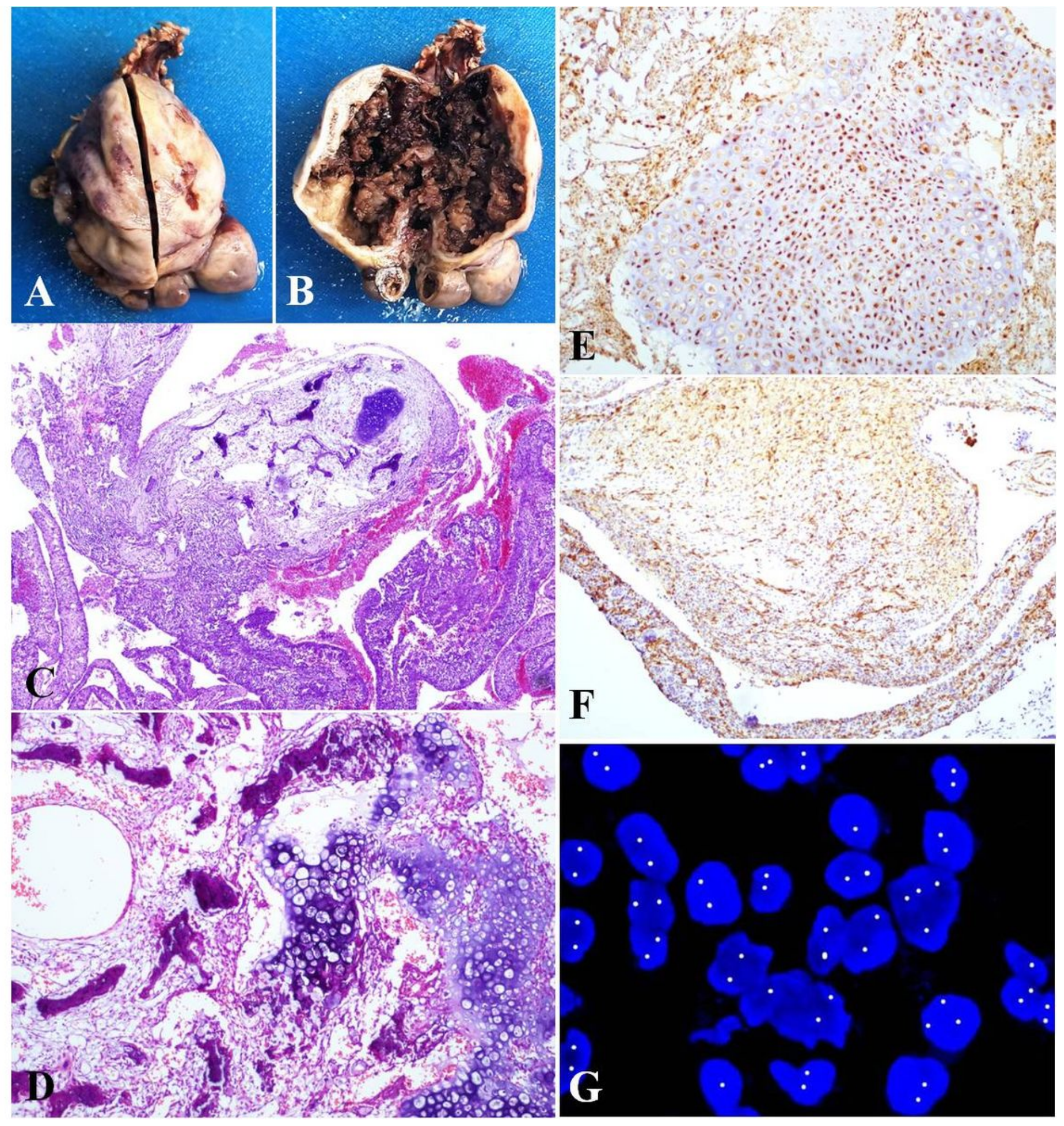

\section{Figure 2}

A The gray-red mass with the 5th rib. B Solid-cystic and multilobulated structures. C The typical features of MHCW, with nodules of cartilage separated by loose fascicles of spindle cells, and cystic changes(x40). D Endochondral calcification, ossification and woven bones(x100). E S-100 was positive for the mature or immature cartilage tissues(x100). F CD34 expressed in the different areas of the ABC- 
like structures $(x 40)$. G FISH showed the negativity of USP6 gene rearrangement by USP6 gene breakapart probe $(x 1000)$.

\section{Supplementary Files}

This is a list of supplementary files associated with this preprint. Click to download.

- renamed951a5.jpg 